ThC1.6

3:00pm-3:15 pm

\title{
Lateral Loss Effects on Modulation Bandwidth in VCSELs
}

P.C. Chui and S.F. Yu

Department of Electrical and Electronic Engineering, University of Hong Kong, Pokfulam Road, Hong Kong.

Summary - Intensity noise and chromatic fiber dispersion in analog optical communication systems are attributed to harmonic and inter-modulation distortion in the amplitude modulation (AM) of semiconductor lasers. In addition, maximum usable bandwidth of the communication systems is limited by harmonic distortion [1]. Due to the use of Fabry Perot type resonator, second harmonic distortion (SHD) exists in both edge-emitting devices and VCSELs. But the distortion caused by lateral loss may be more so in VCSELs because of its smaller core size. Two factors: the lateral field confinement factor $\Gamma$, and the lateral loss $\alpha$ due to diffraction, are considered for their effect on harmonic distortion when the laser device is under small signal modulation.

Modulation causes non-linear variation of carrier concentration $\mathrm{N}$ within the core and the change of $\Gamma$ and $\alpha$ can be modeled by:

$\Gamma\left(\mathrm{N}_{\mathrm{s}}+\Delta \mathrm{N}(\mathrm{t})\right)=\Gamma_{\mathrm{s}}+\frac{\partial \Gamma}{\partial \mathrm{N}} \Delta \mathrm{N}(\mathrm{t}), \quad$ and $\quad \alpha\left(\mathrm{N}_{\mathrm{s}}+\Delta \mathrm{N}(\mathrm{t})\right)=\alpha_{\mathrm{s}}+\frac{\partial \alpha}{\partial \mathrm{N}} \Delta \mathrm{N}(\mathrm{t})$

The steady state $\Gamma_{s}$ and $\alpha_{s}$ are device structure related and vary with the core size of the active region. $\partial \Gamma / \partial \mathrm{N}$ and $\partial \alpha / \partial \mathrm{N}$ are the induced part related to the change of carrier concentration and vary with both the core size and modulation frequency.

The modulation response of VCSEL, taking account into consideration the lateral loss effects $\Gamma, \alpha, \partial \Gamma / \partial \mathrm{N}$ and $\partial \alpha / \partial \mathrm{N}$, can be modeled with a set of rate equations[2]:

$$
\begin{aligned}
& \frac{\partial P(t)}{\partial t}=v_{g}(\Gamma(t) G(t)-\alpha(t)) P(t)+\beta B_{s p} N^{2}(t), \\
& \frac{\partial N(t)}{\partial t}=\frac{I(t)}{q V}-v_{g} \Gamma(t) G(t) P(t)-\frac{N(t)}{\tau_{s}}, \\
& C_{t h} \frac{\partial T(t)}{\partial t}=\left(P_{I V}(t)-P_{h v}(t)\right)-\kappa \frac{T(t)-T_{t}}{R_{t h}}
\end{aligned}
$$

In which the device current $I(t)$, carrier concentration $N(t)$, and photon power $P(t)$ are

obtained by perturbation method: $\quad I(t)=I_{s}+\Delta I(t)=I_{s}+I_{1} e^{j \omega t}$

$$
\begin{aligned}
& N(t)=N_{s}+\Delta N(t)=N_{s}+N_{1} e^{j \omega t}+N_{2} e^{j 2 \omega t} \\
& P(t)=P_{s}+\Delta P(t)=P_{s}+P_{1} e^{j \omega t}+P_{2} e^{j 2 \omega t}
\end{aligned}
$$

Substituting Eqn.(4)-(6) into (1)-(3), the second harmonic component $P_{2}$ and the fundamental component $P_{1}$ can be calculated. The distortion ratio $P_{2} / P_{1}$ can be expressed as:

$$
\frac{P_{2}}{P_{1}}=\frac{\left(2 j \omega+v_{g} \kappa_{s}\right) F(\omega)-v_{g} P_{s} \Gamma_{s} \frac{\partial G_{s f}}{\partial N} E(\omega)}{\left(\omega_{f}^{2}-4 \omega^{2}\right)+2 j \omega v_{g}\left(\kappa_{s}-\Gamma_{s} \frac{\partial G}{\partial P} P_{s}\right)} P_{1} .
$$

where $E(\omega)=v_{g} P_{s} \frac{\partial \Gamma}{\partial N} K(\omega)\left[\frac{\partial G}{\partial P}+\frac{\partial G}{\partial N} K(\omega)\right]+v_{g} \Gamma_{s}\left[\frac{\partial G}{\partial P}+\left(\frac{\partial G_{\text {eft }}}{\partial N}+\frac{1}{\Gamma_{s}} \frac{\partial \alpha}{\partial N}\right) K(\omega)\right]$,

$$
\begin{aligned}
& F(\omega)=v_{g} P_{s} \frac{\partial \Gamma}{\partial N} K(\omega)\left[\frac{\partial G}{\partial P}+\frac{\partial G}{\partial N} K(\omega)\right]+v_{g} \Gamma_{s}\left[\frac{\partial G}{\partial P}+\frac{\partial G_{e f f}}{\partial N} K(\omega)\right], \\
& K(\omega)=\left(v_{g} P_{s} \Gamma_{s} \frac{\partial G_{c f}}{\partial N}\right)^{-1} \times\left(j \omega-v_{g} \Gamma_{s} P_{s} \frac{\partial G}{\partial P}\right), \text { and } \omega_{f}=\text { relaxation oscillation frequency. }
\end{aligned}
$$

For $\omega<<\omega_{\mathrm{f}}$, Eqn.(7) can be approximated by:

$$
\left(\mathrm{P}_{2} / \mathrm{P}_{1}\right) \approx \mathrm{k}(\partial \alpha / \partial \mathrm{N})
$$

where $\mathrm{k}$ is a constant with respect to $\omega$. 
Harmonic distortion within an experimental VCSEL is simulated based on parameter extracted from published data measured for this device[3]. Four sets of data are calculated using our rate equation model. Curves of $P_{2} / P_{1}$ versus modulation frequency are plotted as shown (figure).

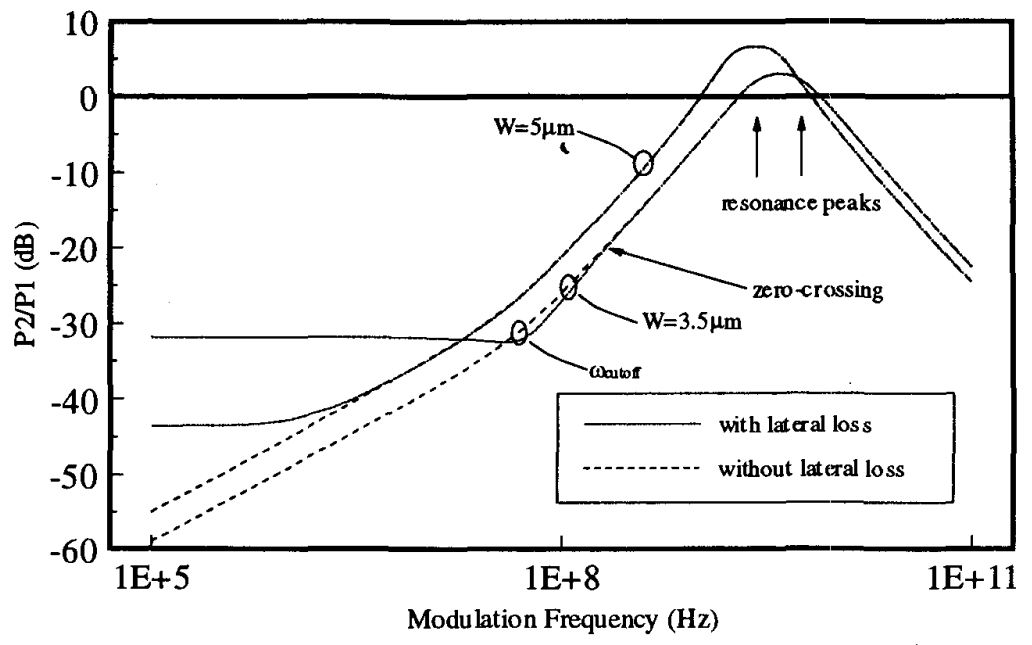

In the presence of lateral loss, $\mathrm{P}_{2} / \mathrm{P}_{1}$ generally assumes a constant level at low modulation frequencies, as predicted by Eqn.(8). When modulation frequency is increased to higher than $\omega_{\text {cutoff }}$, SHD grows. Beyond a zero-crossing point, modulation becomes prohibitive since now the SHD is greater than the fundamental component. The bandwidth for modulation is determined between the cutoff and zero-crossing frequency. The initial constant level of SHD is found to vary with the device's core size. Narrowing the core size shifts both the cutoff and zero-crossing frequencies to the right, widening the modulation bandwidth. Unfortunately this also has the effect of increased SHD in the device. Our data illustrates that at core size $\mathrm{W}=5 \mu \mathrm{m}, \mathrm{SHD}$ is in the -40 to $-50 \mathrm{db}$ level for modulation frequencies up to $\omega_{\text {tutoff }}$ $(\sim 5 \mathrm{MHz})$. Reducing the core size to $\mathrm{W}=3.5 \mu \mathrm{m}$ increases the $\omega_{\text {tutoff }}$ and improves greatly the modulation bandwidth (to $\sim 80 \mathrm{Mhz}$ ), but the minimum SHD level has grown to almost $-30 \mathrm{db}$. By removing the lateral loss, a considerable reduction in SHD is achieved (broken line curves), while $\omega_{\text {tutoff }}$ is not affected, thus a much larger modulation bandwidth is retained.

Our simulation leads to the conclusion that (1) harmonic distortion in VCSEL for frequencies within its modulation bandwidth is directly proportional to $\partial \alpha / \partial \mathrm{N}$. $\partial \Gamma / \partial \mathrm{N}$ has little effect here. (2) a smaller core size design allows for a much improved modulation bandwidth, but the improvement can be nullified by rapidly growing harmonic distortion due to lateral loss effects.

\section{References}

[1] G. Morthier, F. Libbrecht, K. David, P. Vankwikelberge \& G. Baets,'Theoretical investigation of the second order harmonic distortion in the AM response of $1.55 \mu \mathrm{m}$ F-P and DFB lasers', IEEE J. Quantum Electronics, Vol.27, no.8, pp. 1990-2002, 1991.

[2] S.F. Yu, W.N. Wong, P. Shum \& E.H. Li Theoretical analysis of modulation response and second harmonic distortion in vertical cavity surface emitting lasers', IEEE J. Quantum Electronics, Vol.32, no.12, pp. 2139-2147, 1996.

[3] J.W. Scott, R.S. Geels, S.W. Corzine \& L.A. Coldren,'Modeling temperature effects and spatial hole burning to optimize vertical cavity surface emitting laser performance', IEEE J. Quantum Electron., Vol. 29, pp. 1295-1308, 1993. 